\title{
3 Research Square

\section{Toxicity Outcome Using Bladder-Rectum Spacer Balloon Versus Vaginal Gauze Packing in Brachytherapy in Cervical Cancer.}

Nagarjun Ballari ( $\sim$ nagarjunballari@gmail.com )

Post Graduate Institute of Medical Education and Research

\section{Sakshi Rana}

Post Graduate Institute of Medical Education and Research https://orcid.org/0000-0001-7169-1142

Bhavana Rai

Post Graduate Institute of Medical Education and Research

\section{Srinivasa Gowda}

Post Graduate Institute of Medical Education and Research

\section{Suja Bhargavan}

HLL: HLL Lifecare Limited

\section{Raghukumar $\mathbf{P}$}

Regional Cancer Centre Thiruvananthapuram

Abi Santhosh

HLL: HLL Lifecare Limited

\section{Sushmita Ghoshal}

Post Graduate Institute of Medical Education and Research

\section{Research Article}

Keywords: Carcinoma, Brachytherapy, Uterine Neoplasms, Organs at Risk, Radiotherapy

Posted Date: November 30th, 2021

DOI: https://doi.org/10.21203/rs.3.rs-714733/v1

License: (1) (1) This work is licensed under a Creative Commons Attribution 4.0 International License. Read Full License 


\section{Abstract}

\section{BACKGROUND}

To compare the clinical impact in terms of toxicity outcomes with RayshieldTM bladder rectum spacer balloon (BRSB) versus vaginal gauze packing (VGP) in patients treated with high dose rate intracavitary brachytherapy for carcinoma cervix.

\section{RESULTS}

Follow-up and dosimetric data of patients in whom BRSB and VGP were used in a previously reported randomized study were retrieved, 8 . Out of 80 patients analysed, late toxicities assessment (according to Common Terminology Criteria for Adverse Events version 4.0 (CTCAE v4). was possible in 65 patients as 14 patients were lost to follow-up and one patient died. Grade 2 bladder toxicity was observed in 2 patients in each arm. Only 1 patient in VGP arm experienced grade 3 proctitis while none of the patient in BRSB arm had ${ }^{3}$ Grade 2 rectal toxicity. Vaginal toxicity was comparable in both the arms.

\section{CONCLUSION}

No significant difference was observed in bladder and rectal toxicities using the BRSB versus VGP. BRSB can be considered as an alternative to VGP in intracavitary brachytherapy for cervical cancer using tandem- ovoid applicators

The study was approved by the institute ethics committee and registered under Clinical Trial Registry of India (CTRI/2009/091/000840).

\section{Background}

Intracavitary brachytherapy forms an integral part of curative radiotherapy. With brachytherapy, high doses can be delivered to the tumour, but the high dose delivered to adjoining organs at risks (OAR) like rectum, vagina and bladder can result in late toxicity (1). The doses to the organs at risk depend on the patient's anatomy, proper placement of applicator, displacement of OAR's, and stability of the applicator. In high dose rate (HDR) brachytherapy, because of inverse square law, the radiation effects are observed more noteworthy at short distances from the source and less at longer separation(2,3). Hence, in cervical cancer brachytherapy adequate displacement of organs at risk i.e. bladder and rectum and high radiation tolerance of the cervico-uterine complex allows therapeutic doses to be delivered to the target simultaneously respecting the radiation tolerance to the adjoining OARs. Thus a good intra-cavitary application is necessary to achieve the required geometry, the lack of which has been associated with inferior therapeutic outcomes. Displacement of the bladder and/or rectum can be done using various methods like vaginal gauze packing, rectal retractors, posterior vaginal speculum blades, and shielded ovoids $(5,6)$. 
In our previously published experience of using the RayshieldTM bladder rectum spacer balloon (BRSB), the use of BRSB resulted in a statistically significant reduction in rectal $0.1 \mathrm{cc}$ and $2 \mathrm{cc}$ doses on dosimetric analysis $(7,8)$. Although various dosimetric studies have been done using Balloon based system, clinical experiences with respect to bladder and rectal toxicity are lacking. In the present study, we evaluated the outcomes in terms of toxicities comparing the BRSB with VGP.

\section{Methods}

\section{Study design and setting}

This study was a review of follow up and dosimetric data of patients included in our previously published randomized dosimetric BRSB-II study conducted between April 2013-March 2014.7-8 The study was approved by the institute ethics committee and registered under Clinical Trial Registry of India (CTRI/2009/091/000840). The patients were treated with three-dimensional conformal or intensity modulated radiotherapy with a dose of 46 Gy over 4.5 weeks with concurrent cisplatin followed by brachytherapy with 2 fractions of 9 Gy each which were delivered 1 week apart (9). Prior to brachytherapy, the patients were randomized to Vaginal gauze packing (VGP) and the BRSB (Fig. 1). The procedure was performed under general anaesthesia. In all patients, a rectal enema was given before the procedure to ensure complete emptying of the rectum. A reproducible bladder filling protocol was followed before the scan and patient treatment. The bladder was first emptied under gravity and $50 \mathrm{ml}$ saline was inserted in the bladder. It was then allowed to drain freely under gravity. The dose was prescribed to point A (EQD2 $74.5 \mathrm{~Gy}$ ) and the $2 \mathrm{cc}$ doses were evaluated for the bladder, rectum and sigmoid (constraints for bladder D2cc < 90Gy EQD2, rectum < 75Gy EQD2 and sigmoid < 75Gy EQD2).

\section{Follow up}

Patients were followed every 3 months for the first 2 years and 6 months thereafter. Toxicity assessment was done according to $\mathrm{NCl}$ Common Terminology Criteria for Adverse Events version 4.0 (CTCAE v4). The maximum score in any symptom in any of the follow-up visits was considered as the score for statistical analysis. In addition to the bladder and rectal toxicity assessment, vaginal toxicity was also analysed. Since the baseline vaginal length was not available, a subjective assessment was done and any obvious shortening in vaginal length was documented.

\section{Statistics}

Patient details, late toxicities, and dosimetry were entered in SPSS version 19. Descriptive statistics were described for late toxicities and dosimetry. Dosimetry and the incidence of toxicities were compared using paired T-test. A p-value of <.05 implied a significant difference in incidence. Univariate analyses were used to look for co-relations between late toxicities and dosimetric parameters.

\section{Results}


There were 80 patients in which VGP was compared to BRSB. Assessment of late toxicities was possible in 65 patients as 14 patients were lost to follow up and one patient died due to chronic renal failure secondary to diabetes. Out of the total patients, 35 patients were evaluable in BRSB arm and 31 patients in VGP arm (Fig. 2). The mean follow up period was 74.1 months

\section{Rectal toxicity}

There was only one patient out of 31 patients(3.2\%) in VGP arm who experienced grade 3 proctitis. She underwent colonoscopy which showed telangiectasia and to manage the symptoms two applications of Argon plasma coagulation were done. There were no grade 2 toxicities. There were no reported had rectal ulceration, pain, haemorrhage, obstruction, perforation, and fistula in both arms.

\section{Bladder toxicity}

Hematuria and cystitis were seen in both arms.6.8\% $(n=2)$ patient experienced grade2 hematuria in BRSB arm and $6.8 \%(n=2)$ of patient had grade 2 hematuria in VGP arm. Grade 1 Cystitis was seen in 2.8 $\%(n=1)$ of patient in BRSB arm and $6.4 \%(n=2)$ patients experienced grade 1 cystitis in VGP arm. No grade 3 bladder toxicity was seen.

\section{Vaginal toxicity}

In BRSB arm, 31\% $(n=11)$ of patient had vaginal shortening, out of which $11 \%(n=4)$ had grade 1 and 20 $\%(n=7)$ had grade 3 shortening. One patient in both arms had grade 1 vaginal telengectasia. (Table 1$)$. 
Table 1

Late toxicity

\begin{tabular}{|c|c|c|c|}
\hline CATEGORY OF COMPLICATION & BRSB ARM & VGP ARM & P VALUE \\
\hline VAGINAL DRYNESS & 2 & 0 & 0.199 \\
\hline GRADE 1 & 0 & 0 & \\
\hline GRADE 2 & 0 & 0 & \\
\hline \multicolumn{4}{|l|}{ GRADE 3} \\
\hline VAGINAL TELENGECTASIA & 1 & 1 & 0.875 \\
\hline GRADE 1 & 0 & 0 & \\
\hline GRADE 2 & 0 & 0 & \\
\hline \multicolumn{4}{|l|}{ GRADE 3} \\
\hline VAGINAL SHORTENING & 4 & 1 & 0.252 \\
\hline GRADE 1 & 0 & 2 & \\
\hline GRADE 2 & 7 & 4 & \\
\hline \multicolumn{4}{|l|}{ GRADE 3} \\
\hline BLADDER TOXICITY & 1 & 2 & 0.427 \\
\hline CYSTITIS & 0 & 0 & \\
\hline GRADE 1 & 0 & 0 & \\
\hline \multicolumn{4}{|l|}{ GRADE 2} \\
\hline \multicolumn{4}{|l|}{ GRADE 3} \\
\hline HEMATURIA & 0 & 0 & 0.735 \\
\hline GRADE 1 & 2 & 2 & \\
\hline GRADE 2 & 0 & 0 & \\
\hline \multicolumn{4}{|l|}{ GRADE 3} \\
\hline RECTAL TOXICITY PROCTITIS & 0 & 0 & 0.267 \\
\hline GRADE 1 & 0 & 0 & \\
\hline GRADE 2 & 0 & 1 & \\
\hline GRADE 3 & & & \\
\hline
\end{tabular}


EQD2 values of both the groups are shown in Table 2. There was no correlation of EQD2 with grade 2 toxicity.

Table 2

Dosimetry EQD2

\begin{tabular}{|lll|}
\hline OAR & BRSB & VGP \\
\hline BLADDER & $82.8+/-7.6$ & $85.5+/-6.7$ \\
\hline RECTUM & $65.5+/-4.4$ & $69.5+/-4.3$ \\
\hline
\end{tabular}

\section{Discussion}

In the present study, we tried to evaluate the clinical impact of OAR toxicity in patients treated with HDR intracavitary brachytherapy using BRSB and vaginal gauze packing. Numerous studies in the past have compared dosimetric analysis of vaginal gauze packing and different ways of displacing organs at risk, e.g. vaginal packing, intravaginal balloons, rectal retractors $(10,11,12)$. The clinical outcome of these different methods in terms of late toxicity has not been assessed. In our previous dosimetric analysis using the BRSB, a significant reduction in the dose, as well as the inter-fraction dose variation of D2cc of rectum, was observed with the use of BRSB compared to VGP. Although based on the small number of events it does not ascertains the impact of the BRSB, but BRSB and VGB seem to be comparable in terms of OAR toxicity.

As HDR practice over LDR brachytherapy is increasing amongst radiation oncologists, the management of OAR toxicity remains the main goal (13). As the previous studies suggest, the dose delivered to surrounding structures depends upon patient geometry, positional stability of the apparatus, and degree of isodose invasion into critical tissues (14). The skill and expertise of the radiation oncologist and proper applicator placement are critical determinants of dose specification and outcome. In a study by A.N. Viswanathan et al, it was seen that the technical precision of brachytherapy implant placement, especially, the symmetry of the ovoids in relation to the tandem, the displacement of the ovoids in relation to the cervical os, and the appropriateness of packing, affects relapse and disease-free survival rates(15). These parameters should be considered for each brachytherapy implant. Hence, to achieve maximum therapeutic ratio, the dose to the OARs should be minimised by proper displacement in accordance with inverse square law. Studies using the different types of rectum spacers (hydrogel, hyaluronic acid, collagen, biodegradable balloon) in prostate carcinoma have shown a relative reduction in the rectal volume receiving at least $70 \mathrm{~Gy}(\mathrm{~V} 70)$ in the order of $43 \%$ to $84 \%$ and thus reducing rectal toxicities (16). Similarly, reduced rectal doses have been documented with the use of various balloon based systems in cervical cancer brachytherapy.

Despite the evidence that brachytherapy is associated with improved outcomes in cervical cancer, there has been a decline in the use of brachytherapy. Numerous studies have demonstrated that omitting or 
substituting brachytherapy with other options impairs cancer control and survival $(17,18)$. This decline may be a result of the increase use of conformal external beam radiation therapy techniques, most notably intensity modulated radiation therapy (IMRT). The other reason could be its complex procedure which requires training and expertise for optimal application. In a study by Marcrom et al, young residents were more confident in starting an SBRT/SRS practice than a brachytherapy practice, despite SBRT/SRS being a newer treatment technique as brachytherapy requires technical expertise and skill set distinct from that of SBRT/SRS(18). It was believed that trainees can be made expert in brachytherapy with proper training. In case of lack of experience in the initial phases, BRSB could offer an advantage to the learning physicians, decrease the inter-observer variation, and can help in achieving reproducibility. In centers where the procedure is done under sedation or conscious anaesthesia, effective vaginal packing may not be possible because of inadequate relaxation of the patient. In such situations, BRSB offers advantage both in terms of adequate displacement and reproducibility. The other advantages include its CT/MRI compatibility and cost-effectiveness. The cost-effectiveness (BRSB costs around \$13.94(Rs 990)) allows its use even in resource constrained countries which cater to the large burden of cervical cancer. The balloon is reusable and can be used for multiple brachytherapy fractions in a patient. However, the BRSB is limited for use only in the tandem-ovoid applicators and has not been tested for ring applicators. A modification in its design would be hence be required for its wider applicability. In addition, the availability of the balloon in a single size restricts its usage in patients with a narrow vagina, wherein a larger balloon tends to get folded inside the vaginal canal further hindering applicator insertion (7).

\section{Conclusion}

Based on our previous dosimetric results and the clinical data in the present study, RayshieldTM bladder rectum spacer balloon (BRSB) seems to be a propitious alternative to VGB in reducing OAR toxicity. Thus, the BSRB technique may be instrumental in kindling the interest of the radiation oncologists who are preferring IMRT or SBRT, towards brachytherapy as it is simple to use, more consistent, potentially gives lesser complications, and is reproducible in technique, all of which are important in multi-fraction HDR brachytherapy.

\section{Abbreviations}

BRSB BLADDER RECTUM SPACER BALLOON

VGP VAGINAL GAUZE PACKING

NCI CTCAE V4 NATIONAL CANCER INSTITUTE COMMON TERMINOLOGY CRITERIA FOR ADVERSE EVENTS VERSION 4.0

OAR ORGANS AT RISK

HDR HIGH DOSE RATE 
EQD $_{2}$ EQUIVALENT DOSE IN TWO GRAY PER FRACTION

SPSS STATISTICAL PACKAGE FOR THE SOCIAL SCIENCES

IMRT INTENSITY MODULATED RADIATION THERAPY

SBRT STEREOTACTIC BODY RADIOTHERAPY

SRS STEREOTACTIC RADIOSURGERY

\section{Declarations}

\section{Ethics approval and Consent to Participate}

The study was approved by the Post graduate institute of medical education and research "Institute ethics committee" and was registered under Clinical Trial Registry of India (CTRI/2009/091/000840). Written consent for participating in the study was taken from all the participants.

(No specific reference number for ethics committee is applicable)

\section{Consent for publication}

Consent for publication of patient data was taken from all the patients who were part of the study. We confirm that no identifying images or other personal or clinical details of participants are presented that compromise anonymity.

\section{Availability of data and material}

Data is available with the corresponding author

\section{Competing Interests}

The RayshieldTM Bladder-Rectum balloon was provided by HLL life care limited .

Funding_There was no funding for this study.

\section{Authors Contribution}

All authors have read and approved the manuscript.

Conception of Study :BR

Data Analysis : SR and NB

Manuscript Preparation :NB, SR, SG and SGC

Designing of BRSB :AS, PR and SB 
HLL LIFECARE LIMITED.

\section{References}

1. Ferrigno R, dos Santos Novaes PE, Pellizzon AC. etal.High dose-rate brachytherapy in the treatment of uterine cervix cancer. Analysis of dose effectiveness and late complications. Int J Radiat Oncol Biol Phys. 2001;50:1123-35.

2. Katz A, Eifel PJ. Quantification of intracavitary brachytherapy parameters and correlation with outcome in patients with carcinoma of the cervix. Int J Radiat Oncol Biol Phys. 2000;48:1417-25.

3. Nag S, Erickson B, Thomadsen B. etal.The American Brachytherapy Society recommendations for high-dose-rate brachytherapy for carcinoma of the cervix. Int J Radiat Oncol Biol Phys. 2000;48:20111.

4. Bahena JH, Martinez A, Yan D. etal.Spatial reproducibility of the ring and tandem high-dose rate cervix applicator. Int J Radiat Oncol Biol Phys. 1998;41:13-9.

5. Elhanafy OA, Das RK, Paliwal BR. etal.Anatomic variation of prescription points and treatment volume with fractionated high-dose rate gynecological brachytherapy. J Appl Clin Med Phys. 2002;3:1-5.

6. Raghukumar P, Nair RK, Aprem AS. etal.Design and development of an inflatable latex balloon to reduce rectal and bladder doses for patients undergoing high dose rate brachytherapy. J Med Phys. 2009;34:48-51.

7. Rai B, Patel FD, Chakraborty S. etal.Bladder-rectum spacer balloon in high-dose-rate brachytherapy in cervix carcinoma. Int J Radiat Oncol BiolPhys. 2013;85:e217ee222.

8. Rai B, Patel FD, Chakraborty S. Bladder-Rectum Spacer Balloon versus Vaginal Gauze Packing in High Dose Rate Brachytherapy in Cervical Cancer: A Randomised Study (Part II). Clin Oncol (R Coll Radiol). 2015;27:713-9. etal.

9. Patel FD, Rai B, Mallick I. etal.High-dose-rate brachytherapy in uterine cervical cancer. Int J Radiat Oncol Biol Phys. 2005;62:125e130.

10. Eng TY, Patel AJ, Ha CS. Rectal and bladder dose reduction with the addition of intravaginal balloons to vaginal packing in intracavitary brachytherapy for cervical cancer. Brachytherapy. 2016;15:312-8.

11. Rockey WM, Bhatia SK, Jacobson GM. The dosimetric impact of vaginal balloon-packing on intracavitary high dose-rate brachytherapy for gynecological cancer. J ContempBrachytherapy. 2013;1:17-22. etal.

12. Lee $\mathrm{KC}, \mathrm{Kim} \mathrm{TH}$, Choi $\mathrm{JH}$. Use of the rectal retractor to reduce the rectal dose in high dose rate intracavitary brachytherapy for a carcinoma of the uterine cervix. Yonsei Med J. 2004;45:113-22. etal. 
13. MacDonald D, Grigsby P. Comparison of LDR and HDR brachytherapy dose distributions for patients with cervical cancer. Brachytherapy. 2007;6(2):114.

14. Eng T, Fuller $C$, Cavanaugh S. etal.Significant rectal and bladder dose reduction via utilization of foley balloon catheters in high-dose-rate tandem and ovoid intracavitary brachytherapy of the uterine cervix. International Journal of Radiation Oncology*Biology*Physics. 2004;59(1):174-8.

15. Viswanathan A, Moughan J, Small W. etal.The Quality of Cervical Cancer Brachytherapy Implantation and the Impact on Local Recurrence and Disease-Free Survival in Radiation Therapy Oncology Group Prospective Trials 0116 and 0128. International Journal of Gynecologic Cancer. 2012;22(1):123-31.

16. Landwehr N, Georg Krukemeyer M, Wagner W. Can the Rectum Balloon Minimize Rectal Toxicity During Irradiation Therapy of Prostate Cancer? Open Journal of Urology. 2013;03(01):37-43.

17. Haie-Meder C, Kramar A, Lambin P. Analysis of complications in a prospective randomized trial comparing two brachytherapy low dose rates in cervical carcinoma. Int J Radiat Oncol Biol Phys. 1994;29:953-60. etal(.

18. Glaser S, Balasubramani G, Benoit R. etal.The Declining Use of Brachytherapy Boost for Prostate Cancer Despite Associated Survival Advantage. Brachytherapy. 2016;15:23-4.

19. Marcrom,S.,Kahn,J.,Colbert,L.,etal2018.Brachytherapy Training Survey of Radiation Oncology Residents. Brachytherapy,17(4),pp.S25-S26.

\section{Figures}




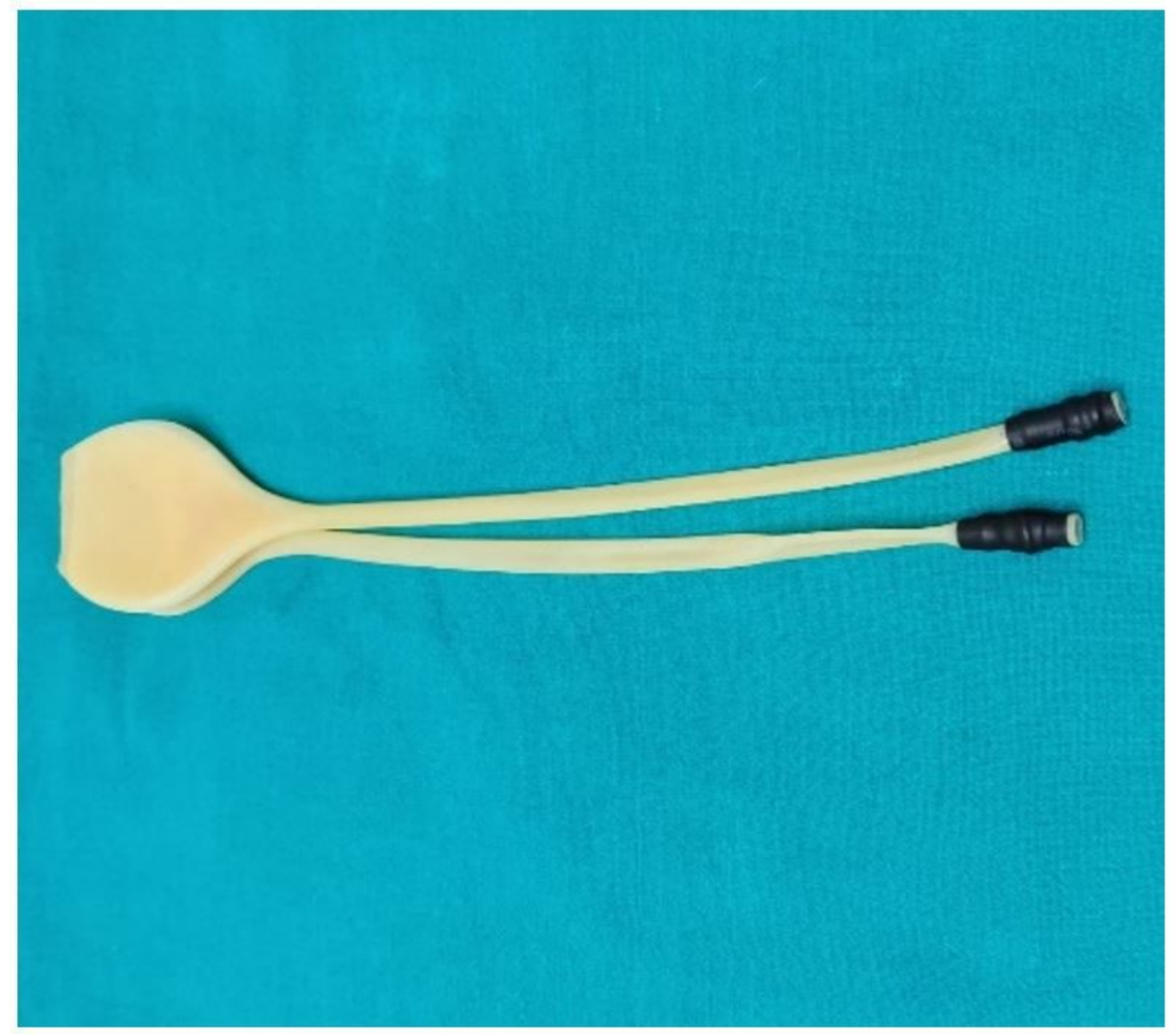

\section{Figure 1}

Figure showing the bladder-rectum spacer balloon with thebladder and rectal part joined together by latex adhesive and joined to a 1-way valve at the tail end of the tubes to prevent the escape of the fluid pushed in. 


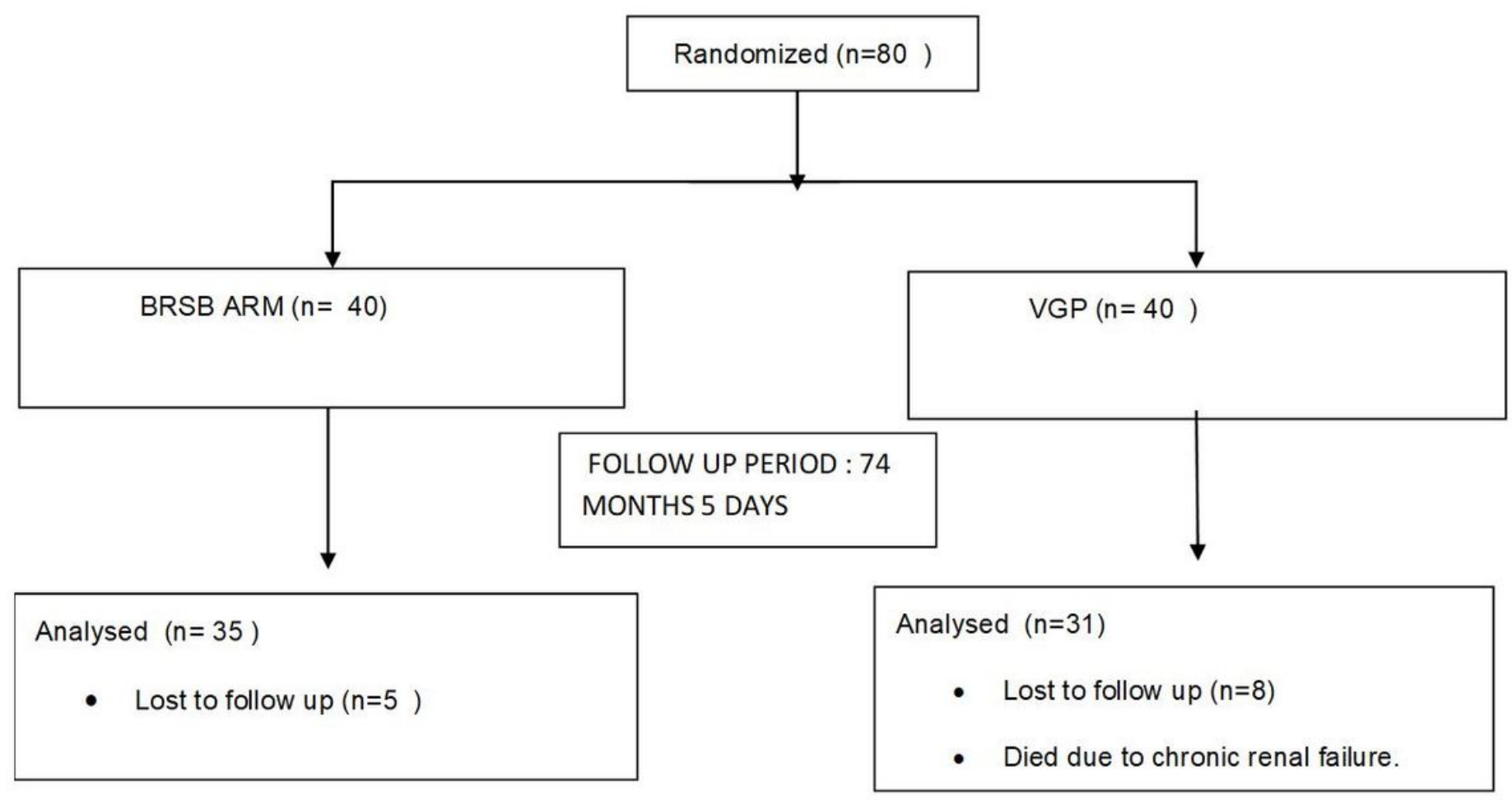

Figure 2

Consort flow chart 